\title{
Retrospective review of ocular alignment after large-angle congenital esotropia surgery
}

\author{
Authors: \\ Roland Höllhumer ${ }^{1}$ \\ Bhavesh Vallabh ${ }^{1}$ \\ Trevor Carmichael ${ }^{1}$

\section{Affiliations:} \\ ${ }^{1}$ Department of \\ Ophthalmology, Division of \\ Neurosciences, University of \\ the Witwatersrand, South \\ Africa \\ Correspondence to: \\ Roland Höllhumer \\ Email: \\ hollhumer@gmail.com \\ Postal address: \\ 19 Cressy Street, Kensington \\ 2094, South Africa

\section{Dates:} \\ Received: 10 Dec. 2014 \\ Accepted: 01 Mar. 2015 \\ Published: 17 July 2015 \\ How to cite this article: \\ Höllhumer R, Vallabh B, \\ Carmichael T. Retrospective \\ review of ocular alignment \\ after large-angle congenital \\ esotropia surgery. Afr Vision \\ Eye Health. 2015;74(1), Art. \\ \#99, 7 pages. http://dx.doi. \\ org/10.4102/aveh.v74i1.99

\section{Copyright:} \\ (C) 2015. The Author(s). \\ Licensee: AOSIS \\ OpenJournals. This work is \\ licensed under the Creative \\ Commons Attribution \\ License.
}

\section{Read online:}

Scan this QR

code with your

smart phone or

mobile device

to read online.
Objective: The study's aims were to evaluate the success of bimedial rectus recession as a primary surgical procedure for patients with congenital esotropia; describe the demographic data of the study group; and compare surgical success with patient age at the time of surgery.

Method: A retrospective review of 52 patients with congenital esotropia $\geq 50 \Delta$ (prism dioptres) was conducted. All patients underwent bimedial rectus recession and were followed-up postoperatively for a minimum of 6 months.

Results: The study period was from January 1992 to September 2003. Fifty-two patients were included in the study group. The pre-operative angle of deviation ranged from $50 \Delta-85 \Delta$. Patient ages ranged from 15 months to 22 years, with a mean of 5.3 years and a median of 4 years. The gender distribution was $42 \%$ male $(n=20)$ and $58 \%$ female $(n=28)$. Surgery was successful (within $10 \Delta$ of orthophoria) in $77 \%(n=40)$, a partial success $(10 \Delta-20 \Delta$ from orthophoria) in $17 \%(n=9)$ and a failure (> 20 $\Delta$ from orthophoria) in $6 \%(n=3)$. No statistically significant relationship was found between surgical success and patient age at the time of surgery.

Conclusion: The study confirmed that bilateral medial rectus recession, performed as a primary procedure for patients with large-angle $(>50 \Delta)$ congenital esotropia, has a high success rate. This finding corresponds with the outcomes of similar international studies.

\section{Introduction}

Esotropias are the most common form of strabismus, making up more than $50 \%$ of paediatric ocular deviations. ${ }^{1,2}$ Congenital esotropias are the most common type of esotropia. The prevalence in the general population of congenital esotropia is approximately $1 \% .{ }^{1}$ In patients with neurological and developmental abnormalities, the prevalence of esotropia may be as high as $30 \% .{ }^{2}$

In patients with congenital esotropia, the deviation is usually more than $35 \Delta$ (prism dioptres) in size, comitant and unchanged between near and far gaze. Seventy-five per cent of patients have an inferior oblique overaction, usually developing by 2 years of age, which produces a V-pattern on examination. Likewise, $75 \%$ of patients may have dissociated vertical deviation. ${ }^{2}$

Before a management plan for a child with esotropia can be instituted, the child needs to undergo a thorough ophthalmic assessment; this involves a complete ocular examination that includes assessing visual acuity, and evaluation of ocular alignment and ocular movements.

All patients require a cycloplegic refraction to exclude any significant refractive errors. A hyperopic refractive error of 1-2 dioptres is normal in young children and commonly found. Patients with accommodative esotropia have an average of 4 dioptres of hyperopia. ${ }^{1}$

Evaluation of ocular alignment starts with observation of the position of a patient's head. Ocular alignment can be assessed by looking at the corneal light reflexes and performing cover tests. There are two tests that make use of the corneal light reflex: the Hirschberg and the Krimsky tests. The cover tests are used to detect horizontal and vertical strabismus; ${ }^{2}$ these include the cover/ uncover test, the alternate cover test and the simultaneous prism and cover test. ${ }^{1,2}$

Ocular movements are evaluated as versions and ductions. ${ }^{2}$ Patients with esotropia may appear to have an abduction deficit owing to cross fixation. This is a phenomenon where the child views the temporal visual field on the one side with the contralateral eye, thereby negating the abduction of the ipsilateral eye and creating an impression of an abduction deficit. ${ }^{1}$

The management of congenital esotropia is mainly surgical. Surgery is only done after reproducible measurements of the ocular deviation have been obtained and any element of amblyopia has 
been treated. Amblyopia can be treated by patching or by pharmacologically penalising the normal eye with atropine eye drops.

Timing of the surgery is an area of great debate, with two main schools of thought. One school advocates early surgery within the first year or even at 6 months of age, the proponents maintaining that there is an improved stereopsis outcome in these patients. The other school of thought advocates a delay in surgery until 2 years of age, when any inferior oblique overaction and dissociated vertical deviation should be apparent. Delaying esotropia surgery allows these associated deviations to become manifest and to be corrected in one operation. ${ }^{2}$ Small-angle esotropias may resolve spontaneously in the first 6 months of life. The Congenital Esotropia Observational Study showed that stable angles of more than $40 \Delta$ are unlikely to resolve spontaneously, and these patients are therefore candidates for early surgery. ${ }^{3}$

There are two main surgical approaches: (1) operations on both eyes, with bilateral medial rectus recessions and possible lateral rectus resections; or (2) surgery on only one eye comprising a medial rectus recession and a lateral rectus resection. ${ }^{2}$ At St John Eye Hospital (St John), Johannesburg, patients with untreatable or significant residual amblyopia in one eye usually follow the second surgical approach (surgery involving only the amblyopic eye) whilst other patients follow the first. The benefit of bimedial rectus recession is that it is a faster procedure and leaves the lateral rectus untouched if the need for subsequent surgery arises. ${ }^{4}$

The amount of recession or resection of the muscles is based on the Calhoun algorithm: each millimetre that the medial rectus is recessed is equivalent to $4 \Delta$ of alignment change, and each millimetre of resection of the lateral rectus is equivalent to a $2 \Delta$ change in alignment. ${ }^{5}$ It was previously thought that inserting muscles behind the equator would cause a limitation of ocular movement. However, it has been shown in various studies that this is not the case, thus allowing a muscle to be recessed by a greater extent. ${ }^{6}$ At St John, the maximal medial rectus recession performed is $7.5 \mathrm{~mm}$ and the maximal lateral rectus resection performed is $10 \mathrm{~mm} .{ }^{5}$ It has been suggested that an alignment within $10 \Delta$ of orthophoria at the 6-month follow-up can be considered a surgical success. ${ }^{4,6}$

Forrest et al. ${ }^{4}$ did a retrospective study of 49 patients with

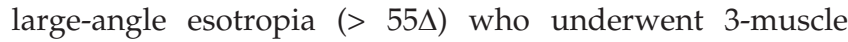
surgery, consisting of a bimedial rectus recession and lateral rectus resection. They had a success rate of $91 \%$ at the 6-month follow-up.

Thomas et $a .^{6}$ did a retrospective study on the success of a single surgical procedure, including 2/3/4-muscle surgery, for large-angle (> 50 $\Delta$ ) strabismus. The esotropia arm had an overall success rate of $69 \%$ with a mean follow-up time of 4.7 months.

Damanakis et al. ${ }^{7}$ reviewed the success of bilateral $8 \mathrm{~mm}$ medial rectus recessions in 16 patients. They found that
$75 \%$ of the procedures were successful, whilst $25 \%$ were undercorrected and required further lateral rectus surgery. None of the patients was overcorrected or had adduction deficits postoperatively.

Vroman et al. ${ }^{8}$ conducted a retrospective analysis of patients with congenital esotropia, by comparing the success of 2 -muscle surgery in patients with esotropia $<50 \Delta$ with 2 -muscle surgery for esotropia $>50 \Delta$. The recession for the smaller angles was based on a surgical dosage table. The bimedial rectus recessions dosage used for large angles was $6.5 \mathrm{~mm}$ for $55 \Delta-60 \Delta$ and $7 \mathrm{~mm}$ for $>65 \Delta$. The mean followup time was 32 months for the large-angle group. Sixteen patients were assigned to the large-angle group (> 50 $\Delta$ esotropia) and had a $75 \%$ success rate. None of the patients developed a consecutive exotropia.

Rowe et al. ${ }^{9}$ looked at children with esotropia who underwent surgical correction before 2 years of age and were followedup until at least 4 years of age. The patients underwent either bimedial rectus recession or a unilateral medial rectus recession with a lateral rectus resection. When examining the results, 24 of the 40 study patients had an esotropia $>50 \Delta$. Eight of the 24 underwent a recess-resect procedure with a $38 \%$ success rate. The rest of the patients underwent bimedial rectus recession with a $31 \%$ success rate.

Szmyd et al. ${ }^{10}$ held a retrospective review of 45 patients with a congenital esotropia $>50 \Delta$ who underwent bimedial rectus recessions of $6 \mathrm{~mm}-7 \mathrm{~mm}$. Thirty-seven of the patients had deviations from $50 \Delta-65 \Delta$ and underwent $6 \mathrm{~mm}$ recessions. Eight of the patients had deviations $>70 \Delta$ and underwent $7 \mathrm{~mm}$ recessions. There was an $89 \%$ success rate in the first group and a $100 \%$ success rate in the latter group. The overall success for bimedial rectus recessions in this large-angle esotropia study was $91 \%$.

Nelson et al. ${ }^{11}$ reviewed the records of 97 patients with congenital esotropia $>50 \Delta$ who were followed-up for more than 6 months postoperatively. Eighty-eight of the patients had deviations of $50 \Delta-70 \Delta$ and underwent $6 \mathrm{~mm}$ bimedial rectus recession. The remaining patients had deviations $>70 \Delta$ and had $7 \mathrm{~mm}$ bimedial rectus recession. The first group had a success rate of $83 \%$ and the second group of $89 \%$ at 6 months postoperatively. The overall success rate was $83.5 \%$.

Weakley et al..$^{12}$ reviewed the surgical records of 36 patients with an infantile esotropia $>60 \Delta$ who underwent bilateral $7 \mathrm{~mm}$ medial rectus recession. They had an $85 \%$ success rate at 6 months postoperatively; this rate dropped to $75 \%$ with a longer follow-up (mean 18.2 months). This change was mostly attributed to the late onset of an accommodative element that worsened a previously corrected esotropia. The authors also found that ocular alignment 6 weeks after the operation had a high predictive value for final outcome.

Scott et al. ${ }^{13}$ performed a retrospective review of patients who underwent 2-muscle surgery or more than 2-muscle surgery 
for congenital esotropia $>50 \Delta$. They reviewed 59 patients who underwent 2-muscle surgery, of either a bimedial rectus recession $(n=57)$ or unilateral medial rectus recession with lateral rectus resection $(n=2)$. The bimedial recessions ranged from $5 \mathrm{~mm}-6.5 \mathrm{~mm}$, which is less than the current maximum of $7.5 \mathrm{~mm}$. They had surgical success in only $37 \%$ of the patients, whilst $58 \%$ were undercorrected.

Lee et al. ${ }^{14}$ did a retrospective review of congenital esotropia patients with an angle $>50 \Delta$. They performed bimedial rectus recessions of $4.5 \mathrm{~mm}-5 \mathrm{~mm}$ together with bilateral lateral rectus resections of $4 \mathrm{~mm}-8 \mathrm{~mm}$. They had a $61 \%$ success rate with this approach, whilst the other patients required a second and even a third procedure. The patients who required further surgery were an average of 11 months younger than the successful group.

Hess et al. ${ }^{15}$ reviewed patients with congenital esotropia $>35 \Delta$ who underwent a graded bimedial rectus recession. There were 11 patients with an angle of deviation $>60 \Delta$ who underwent recessions of $7 \mathrm{~mm}-8 \mathrm{~mm}$. The success rate was $55 \%$.

There is a paucity of local studies evaluating the outcome of strabismus surgery performed in South Africa. State hospitals are burdened by a large workload, and it follows that the reduction of secondary strabismus surgeries in patients with large-angle congenital esotropia would significantly help to maximise the efficient use of theatre time. It will be useful to review the records of large-angle esotropia surgery at our own institution to assess the success of surgical alignment.

\section{Materials and methods}

The present work is a retrospective study of 52 patients with large-angle congenital esotropia who were operated on at St John. For the purpose of the study, a large-angle esotropia was defined as an angle $>50 \Delta$ in primary gaze. The sample size was chosen based on the literature review, in which the studies $^{6,7,8}$ used a sample size of 50 patients and were able to achieve statistically significant results. The study was registered with and approved by the Human Ethics Research Committee of the University of the Witwatersrand.

All patients were fully evaluated by ophthalmology registrars to exclude any underlying systemic, neurological or ocular diseases. The patients were then referred to the resident optometrist for a cycloplegic refraction to exclude any significant refractive error. A refractive error $>3$ dioptres from emmetropia was considered to be significant. ${ }^{1}$ If the preceding examinations revealed no abnormalities other than the underlying squint, the patients were referred to the resident orthoptist for a comprehensive examination of ocular motility and alignment. The orthoptist made use of the cover/uncover test, alternate cover test, Krimsky test and/or the Hirschberg test to evaluate the angle of ocular deviation. This angle was then entered into the orthoptist's register as the pre-operative angle of esotropia. If more than one assessment was done, the final accepted pre-operative assessment was entered into the register. If amblyopia was found, this was managed with patching or penalising with atropine. If amblyopia was absent and measurement of the deviation was constant, the patients were referred for surgery and operated on by consultants and registrars at St John. The following alignment algorithm was used: each millimetre that the medial rectus is recessed is equivalent to $4 \Delta$ of alignment change, and each millimetre of resection of the lateral rectus is equivalent to $2 \Delta$ change in alignment. ${ }^{5}$

The patients were reviewed postoperatively at day one by the surgeon responsible for the surgery. If the surgery was uneventful, the patient was discharged and reviewed within a month at the paediatric ophthalmology clinic. At this later visit, the patient was seen by both the orthoptist and doctors in the clinic; the orthoptist's register was updated regarding postoperative ocular alignment; and any abnormalities in ocular motility were noted. Following this visit, patients were seen at various intervals (usually after 6 weeks, 3 months and 6 months), and the register updated at each visit.

The orthoptist records were used to complete the data capture sheets. Data were collected from the register between January 1992 and September 2003. The patients were chosen solely on the diagnosis of a congenital esotropia measuring $\geq 50 \Delta$ in size. Surgery was regarded as successful if the postoperative deviation was within $10 \Delta$ of orthophoria (esotropia or exotropia), a partial success if the postoperative deviation was between $10 \Delta$ and $20 \Delta$, and a failure if the postoperative deviation was $>20 \Delta$ from orthophoria at 6 months or longer after surgery. ${ }^{4}$

\section{The inclusion criteria were:}

- onset of esotropia before 6 months of age

- only patients where a bimedial rectus recession had been performed

- at least 6 months postoperative follow-up.

The exclusion criteria were:

- neurological abnormalities

- amblyopia

- developmental delays

- nystagmus

- refractive errors $>3$ dioptres from emmetropia

- history of botulinum toxin injections or previous squint surgery.

At times it may be difficult to assess amblyopia in young children, and this was a relative exclusion criterion.

The captured data were analysed with the following outcome measures in mind:

- objective 1: description of the patient demographics, including age, gender and race

- objective 2: determination of the rate of success, partial success and failure of surgery

- objective 3: comparison of the success of surgery with patient age at the time of surgery 
- objective 4: to review the rate of re-operation that would be needed in the groups with partial success and failure of surgery.

Complications that occurred during and after surgery were not recorded in the orthoptist's register, and were therefore not included as an objective in the present study.

The hypothesis of the study is that the surgery results at St John compare favourably with results in the literature.

Data were entered from the orthoptist's register onto data capture forms. Patients with incomplete data sheets were excluded from the study.

The demographics in objective 1 recorded the following: (1) age is a continuous variable, and the range, mean and median were described for the total group of patients and for the three subgroups (success, partial success and failure), (2) gender is a categorical variable, and the frequency was described for the total group and the three subgroups of patients and (3) race is also a categorical variable, and its frequency was described for the total group as well as the three subgroups.

For objective 2, the variables are categorical and the frequencies were calculated for each of the three subgroups (success, partial success and failure).

Objective 3 compared the success of surgery with the age of the patient at the time of surgery; that is, compares a categorical variable with a continuous variable. Owing to the lack of normality in the age distribution, the Kruskal-Wallis formula was used to compare the statistical significance of these two variables. The test was done at an alpha of $5 \%(p \leq 0.05)$.

Objective 4 evaluated the rate of re-operation needed, which is a categorical variable. The frequency was calculated.

Stata version 13.1 computer software was used for the statistical analysis.

\section{Ethical consideration}

The study was approved by the Human Ethics Research Committee (Medical) of the University of the Witwatersrand. The certificate clearance number was M130943.

\section{Results}

The study period was from January 1992 to September 2003. Fifty-two patients from a potential 475 patients met the inclusion criteria for the study. The main reason for patients to be excluded was that at least 6 months of postoperative follow-up had not been documented. The pre-operative angle of deviation ranged from $50 \Delta-85 \Delta$ of esotropia. Table 1 demonstrate the distribution of pre-operative deviation. The angle of deviation in most patients ranged from $50 \Delta-65 \Delta$ $(n=44)$, with only 7 patients having a deviation $>70 \Delta$ and only 1 patient $>80 \Delta$.
The patients ranged in age from 15 months to 22 years, with a mean of 5.3 years and median of 4 years. Table 2 demonstrates the age distribution. As can be seen, it is skewed with most patients being under 10 years old.

In the study group, there was a slight female preponderance of $58 \%(n=28)$, whilst $42 \%(n=20)$ of the patients were male, as shown in Table 3. All patients in the study were black individuals $(n=48)$. The gender and race classifications of 4 patients were not documented in the register.

The surgical success rate was $77 \%(n=40)$, as shown in Table 4. Ages in this group ranged from 15 months to 18 years (mean of 5 years and median 4 years), gender distribution was $44 \%(n=16)$ male and $56 \%(n=20)$ female, and demographic data were not available for 4 patients. Four patients had a consecutive exotropia up to $8 \Delta, 33$ patients had a residual esotropia up to $10 \Delta$, and 3 patients were completely orthophoric at the 6-month follow-up.

The partial success rate was $17 \%(n=9)$. Sixty-seven per cent $(n=6)$ were undercorrected, whilst 33\% $(n=3)$ had a consecutive exotropia ranging from $12 \Delta-16 \Delta$. The ages in the partial success group ranged from 2 to 6 years, with a mean

TABLE 1: Distribution of pre-operative angle of deviation as number and percentage of the total of 52 patients.

\begin{tabular}{lcc}
\hline Angle of Esotropia & Number of patients & Percentage \\
\hline $50 \Delta$ & 13 & 25 \\
$55 \Delta$ & 12 & 23 \\
$60 \Delta$ & 10 & 19 \\
$65 \Delta$ & 9 & 17 \\
$70 \Delta$ & 4 & 8 \\
$75 \Delta$ & 3 & 6 \\
$80 \Delta$ & 1 & 2 \\
\hline
\end{tabular}

TABLE 2: Age distribution versus number and percentage for those patients with known demographic data.

\begin{tabular}{lcc}
\hline Age of patient at surgery & Number of patients & Percentage \\
\hline 16 Months & 1 & 2 \\
18 Months & 2 & 4 \\
2 Years & 5 & 9 \\
3 Years & 13 & 25 \\
4 Years & 8 & 15 \\
5 Years & 7 & 13 \\
6 Years & 4 & 8 \\
7 Years & 2 & 4 \\
8 Years & 3 & 6 \\
9 Years & 2 & 4 \\
11 Years & 2 & 6 \\
12 Years & 1 & 2 \\
18 Years & 1 & 2 \\
22 Years & 1 & 2 \\
\hline$N=48$. & &
\end{tabular}

TABLE 3: Gender distribution as numbers and percentages of those patients with known demographic data.

\begin{tabular}{lcc}
\hline Gender & Number of patients & Percentage \\
\hline Male & 20 & 42 \\
Female & 28 & 58 \\
\hline
\end{tabular}

$N=48$. 
TABLE 4: Surgical results in three subgroups (successful, partial success and failure) as numbers and percentages of the total study group.

\begin{tabular}{lcc}
\hline Surgical outcome & Number of patients & Percentage \\
\hline Successful & 40 & 77 \\
Partial success & 9 & 17 \\
Failure & 3 & 6 \\
\hline
\end{tabular}

$N=52$.

TABLE 5: Postoperative follow-up periods of study patients in percentages.

\begin{tabular}{lcc}
\hline Follow-up periods & Number of patients & Percentage \\
\hline 6-12 Months & 19 & 37 \\
$13-18$ Months & 11 & 21 \\
$19-24$ Months & 9 & 17 \\
$25-48$ Months & 10 & 19 \\
$49-76$ Months & 3 & 6 \\
\hline
\end{tabular}

$N=52$.

of 4.1 years and a median of 4 years. The gender distribution was $22 \%(n=2)$ male and $78 \%(n=7)$ female.

The failure rate was $6 \%(n=3)$. These were all unsuccessful owing to undercorrection, with a residual esotropia ranging from $30 \Delta-75 \Delta$. The ages in this group ranged from 8 years to 22 years, with a mean of 12.7 years and a median of 8 years. The gender distribution was $67 \%(n=2)$ male and $33 \%(n=1)$ female.

Age at the time of operation was evaluated for normality using the Shapiro-Wilk test. It was found that the data did not have a normal distribution. For this reason, the KruskallWallis test was used to compare age of patients at surgery with surgical outcome, and showed an almost statistically significant relationship between age and surgical success, with $p=0.06$. However, this figure does not meet the criteria for statistical significance of $p \leq 0.05$.

The follow-up period ranged from 6 months to 6 years and 4 months, with a mean of 21 months. Table 5 demonstrates the distribution of the follow-up period. Most patients were followed-up for $\leq 20$ months.

No patients were recorded as having had a second procedure.

\section{Discussion}

The present study demonstrates a good surgical outcome for patients with a large-angle esotropia undergoing bimedial rectus recession as a primary procedure. The success rate of $77 \%$ compares favourably with other studies in the literature.

Damanakis et al. ${ }^{7}$ reviewed 16 patients with a congenital esotropia of $80 \Delta-90 \Delta$ who underwent $8 \mathrm{~mm}$ bimedial rectus recession. They showed a success rate of $75 \%$. The maximal recession done in the present study was $7.5 \mathrm{~mm}$, showing similar results. Damanakis's study may indicate that, for extremely large deviations $(80 \Delta-90 \Delta)$, larger recessions than those performed at St John may be needed to achieve similar results. However, the present study had only one patient with an angle of deviation $>80 \Delta$ who had a successful outcome.
Vroman et al. ${ }^{8}$ reviewed patients with congenital esotropia undergoing surgery. Patients with deviations > $>\Delta 0 \Delta$ underwent a $6.5 \mathrm{~mm}-7 \mathrm{~mm}$ bimedial rectus recession with a success rate of $75 \%$. In the present study, we generally used a slightly larger maximal recession of $7.5 \mathrm{~mm}$, but showed similar results.

Weakley et al. ${ }^{12}$ reviewed the surgical results of patients with deviations $>60 \Delta$ undergoing $7 \mathrm{~mm}$ bimedial rectus recessions. Their early results showed an $85 \%$ success rate (at 6 months); however, this decreased to $75 \%$ at longer follow-up (18 months). The late failures were attributed to an accommodative element. The present study followedup patients for at least 6 months. The number of patients requiring spectacles at a later date was only 5 . All children who needed spectacles were $\geq 3$ years old and none was a surgical failure with their spectacle correction. This number may, however, be skewed owing to follow-up failure after 6 months in many patients. It is therefore important to bear the accommodative element in mind in patients with lateonset failures after initially successful surgery. Weakley et al. additionally found that the 6-week postoperative follow-up was a good predictor of final outcome. In the present study, although all patients were followed-up for at least 6 months, the follow-up was sometimes haphazard, and only 15 of the 52 patients had a follow-up at the 6-week postoperative interval. Of those followed-up patients, 11 were a surgical success at 6 weeks and 10 remained a surgical success at the last follow-up, whilst 1 patient became a partial success. Three of the patients were a partial success at 6 weeks, with 1 becoming successful and the other 2 becoming failures at the last visit. There was 1 failure at the 6-week follow-up who remained so at the final follow-up. These outcomes seem to support Weakely et al. ${ }^{12}$ in that almost all the successes at 6 weeks were successful at the last follow-up. The partial successes and failures tended to remain so or worsen with time; but they were a small sample in our study.

Scott et al. ${ }^{13}$ reviewed patients undergoing 2-muscle surgery for large-angle $(>50 \Delta$ ) congenital esotropia. Fifty-seven of these patients had bimedial rectus recessions of only $5 \mathrm{~mm}-$ $6.5 \mathrm{~mm}$ - less than the standard amount. They achieved a success rate of only $37 \%$, which supports our practice of larger recessions in patients with larger angles of deviation. As done at St John, each millimetre of medial rectus recession equates to a $4 \Delta$ decrease in esotropia, so a deviation of $50 \Delta$ would have a recession of $6 \mathrm{~mm}$ as a minimum primary procedure, rather than the $5 \mathrm{~mm}$ used by Scott et al.

The present study therefore correlates well with the current literature in supporting large bimedial rectus recessions for large-angle congenital esotropia. The present study had a success rate of $77 \%$, partial success rate of $17 \%$ and failure rate of $6 \%$. Seven patients $(13 \%)$ of the total of 52 were overcorrected and had consecutive exotropia. Of the 7 overcorrections, 4 had an overcorrected angle within $10 \Delta$ of orthophoria and were considered a surgical success and not a significant overcorrection. The 3 other overcorrected patients 
formed part of the partial success group as their consecutive exotropia measured between $10 \Delta$ and $20 \Delta$. The first patient had a deviation of $50 \Delta$ but had a bimedial rectus recession of $6.5 \mathrm{~mm}$. According to the surgery table, this deviation warranted a $6 \mathrm{~mm}$ bimedial rectus recession, equating to a $24 \Delta$ exotropic shift per muscle. The second patient had a deviation of $55 \Delta$ and had bimedial rectus recessions of $7 \mathrm{~mm}$, again too large according to the surgical tables. The third patient had a deviation of $50 \Delta-55 \Delta$ and had bimedial rectus recessions of $6 \mathrm{~mm}$, which was appropriate surgery for this patient, who had a $14 \Delta$ exotropia at 2 years' follow-up. The rest of the patients in the partial success group were undercorrected with a residual esotropic deviation of $10 \Delta-20 \Delta$.

In the failed surgery group, there were no overcorrections, only undercorrections. The first patient had a pre-operative deviation of $75 \Delta$. The patient had only $7 \mathrm{~mm}$ recessions, which is inadequate according to the surgical tables. The patient had a good postoperative angle of $6 \Delta$, but deteriorated to a deviation of $30 \Delta$ esotropia at the 6-month follow-up. This patient did not require spectacles. The second failure had a pre-operative deviation of $60 \Delta$ and underwent $7 \mathrm{~mm}$ recessions, which was appropriate. The early postoperative deviation of $14 \Delta$ esotropia, would generally be a good indicator of orthophoria later on. However, at the 6-month follow-up, the deviation was $70 \Delta$ esotropia, larger than the pre-operative deviation of $60 \Delta$. No record was made of a subsequent procedure. The last failure had a pre-operative deviation of $70 \Delta-75 \Delta$ and underwent $7 \mathrm{~mm}$ recessions, which was inadequate according to the surgical tables. The postoperative deviation was $45 \Delta$ esotropia that persisted at the 10-month follow-up.

A limitation of the present study that must be kept in mind is the varying levels of expertise of the surgeons involved, who were registrars and consultants. Registrars were supervised according to their level of seniority, which might have had an effect on the success rate. However, with a success rate comparable to international studies, this factor might have had less of an effect than expected. What probably played a more important role was the pre-operative measurement and correct calculation of the amount of recession required. As seen with the partial success group, incorrect preoperative calculations were done for 2 of the 3 patients who were overcorrected. A further limitation is the absence of records for re-operation. It would be assumed that patients in the partial and unsuccessful groups would have undergone further procedures, but this was not recorded in the orthoptist's register. The rate of secondary procedures needed therefore would be $23 \%(n=12)$. This figure does not affect the present study significantly, as the primary objective was to assess the success of primary surgery.

A selection bias is also inherent in the present study's design. Finding cases that fulfil all the inclusion and exclusion criteria will garner a patient group with improved compliance and follow-up. In the present study, that effect might have translated into better pre-operative measurement and better postoperative follow-up, and therefore result in better outcomes; but in fact, only 52 patients out of 475 patients who underwent bimedial rectus recession for congenital esotropia fulfilled the inclusion criteria, mostly owing to inadequate follow-up.

The Kruskall-Wallis test was done to compare success rates in relation to age of patient at the time of surgery. The test showed no statistically significant difference in the success rates for different age groups, with a confidence interval of $p=0.06$.

Although not an objective of the study, it is interesting to look at the surgical dosages used and relate them to the surgical outcome. The study group was too small to formally draw any conclusions, but patients who had the appropriate surgical dosage in the $50 \Delta$ and $60 \Delta$ group had a higher success rate. It would be worthwhile to carry out a future study designed and powered to specifically compare the preoperative angle of deviation with surgical dosage and postoperative alignment outcomes.

\section{Conclusion}

Congenital esotropia is the most common form of esotropia seen at St John Eye Hospital, accounting for the vast majority of squint surgery performed at the institution. The present study confirms the validity of bimedial rectus recession as a primary procedure with a high success rate for congenital esotropias exceeding $50 \Delta$.

\section{Acknowledgements}

We gratefully acknowledge the assistance of Professor T.R. Carmichael (Head of Department of Ophthalmology, University of the Witwatersrand); resident orthoptists at St John Eye Hospital; and personnel of the Department of Statistics, University of the Witwatersrand.

\section{Competing interests}

The authors declare that they have no financial or personal relationships which may have inappropriately influenced them in writing this article.

\section{Authors' contributions}

The study was conducted by R.H. (University of the Witwatersrand) and supervised by B.V. (University of the Witwatersrand) and T.C. (University of the Witwatersrand).

\section{References}

1. Diamond GR. Paediatric and adult strabismus. In: Yanoff M, Duker JS, editors. Ophthalmology. 3rd ed. St. Louis: Mosby, 2008; p. 580-634.

2. Raab EL, Aaby AA, Bloom JN, et al. Pediatric ophthalmology and strabismus. 2011-2012 ed. San Francisco: American Academy of Ophthalmology; 2012.

3. Hutcheson KA. Childhood esotropia. Curr Opin Ophthalmol. 2004;15:444-448. http://dx.doi.org/10.1097/01.icu.0000135505.89223.f6

4. Forrest MP, Finnigan S, Finnigan S, Gole GA. Three horizontal muscle squint surgery for large angle infantile esotropia. Clin Experiment Ophthalmol. 2003;31:509-516. http://dx.doi.org/10.1046/j.1442-9071.2003.00713.x

5. Calhoun J. Extra-ocular muscle surgery techniques. In: McAllister L, editor. Atlas of pediatric ophthalmology surgery. Toronto: WB Saunders, 1987; p. 1-21. 
6. Thomas S, Guha S. Large-angle strabismus: Can a single surgical procedure achieve a successful outcome? Strabismus. 2010;18:129-136. http://dx.doi.org/10.3109/ 09273972.2010.525778

7. Damanakis AG, Arvanitis PG, Ladas ID, Theodossiadis GP. $8 \mathrm{~mm}$ bimedial rectus recession in infantile esotropia of $80-90$ prism dioptres. $\mathrm{Br} J$ Ophthalmol. 1994;78:842-844. http://dx.doi.org/10.1136/bjo.78.11.842

8. Vroman DT, Hutchinson AK, Saunders RA, Wilson ME. Two-muscle surgery for congenital esotropia: rate of reoperation in patients with small versus large angles of deviation. J AAPOS. 2000;4:267-270. http://dx.doi.org/10.1067/mpa. 2000.106960

9. Rowe FJ. Long-term postoperative stability in infantile esotropia. Strabismus 2000;8:3-13. http://dx.doi.org/10.1076/0927-3972(200003)8:1;1-6;FT003

10. Szmyd SM, Nelson LB, Calhoun JH, Spratt C. Large bimedial rectus recessions in congenital esotropia. Br J Ophthalmol. 1985;69:271-274. http://dx.doi.org/ 10.1136/bjo.69.4.271
11. Nelson LB, Calhoun JH, Simon JW, Wilson T, Harley RD. Surgical management of large angle congenital esotropia. Br J Ophthalmol. 1987;71:380-383. http:// dx.doi.org/10.1136/bjo.71.5.380

12. Weakley DR Jr, Stager DR, Everett ME. Seven-millimeter bilateral medial rectus recessions in infantile esotropia. J Pediatr Ophthalmol Strabismus. 1991;28: 113-115.

13. Scott WE, Reese PD, Hirsch CR, Flabetich CA. Surgery for large-angle congenital esotropia. Two vs three and four horizontal muscles. Arch Ophthalmol. 1986;104:374-377. http://dx.doi.org/10.1001/archopht.1986. 01050150074030

14. Lee DA, Dyer JA. Bilateral medial rectus muscle recession and lateral rectus muscle resection in the treatment of congenital esotropia. Am J Ophthalmol. 1983;95:528-535. http://dx.doi.org/10.1016/0002-9394(83)90276-3

15. Hess JB, Calhoun JH. A new rationale for the management of large angle esotropia. J Pediatr Ophthalmol Strabismus. 1979;16:345-348. 